\title{
The Information System by Uncertainty Theory
}

\author{
M. Shokry**, Manar Omran* \\ Department of Physics and Engineering Mathematics, Tanta University, Egypt \\ Email: **mohnayle@yahoo.com, . omranmanar900@yahoo.com
}

\begin{abstract}
The theories of uncertainty are very useful to treat with mathematics that needs to address and in providing a flexible model to elaborate uncertainty and vagueness involved in decision making. In this paper, we used the information system by converting the information system to the triangular fuzzy number matrix; into its membership function and by computing the relation matrices to take the suitable decision with degree. In this paper, we also introduced an application in real life.
\end{abstract}

Keywords- Fuzzy matrices, Information system, triangular fuzzy number, Horizontal Curve Design, membership function.

\section{INTRODUCTION}

By developing intelligent systems, we can operate with real life and engineering problems. A large part of mathematics is based on the notions of a set .Set theory, however, was founded by a single paper in 1874 by George Cantor " on a property of the collection of all real algebraic numbers"[1]. In classical set theory.an element either belongs to a set or does not belong to a set .Fuzziness [2] plays an essential role in human life because most of the classes encountered in the real physical world are fuzzy. In 1965, Zabeh [3] introduced the idea of a fuzzy set as an extension of the classical set theory. The idea of intuitionistic fuzzy set" was first published by krassimir $\mathrm{T}$. Atanassov [4] and many works by the same author and his colleagues appeared in the literature [5], [6], [7]. Established by Florentin smarandache in 1980 ,neurosophy was presented as the study of the origin, nature, and scope of neutralities, as well as their interactions with different ideational spectra The main Idea was to consider an entity ,"A" in relation to its opposite "Non-A , and to that which is neither "A" nor Non - A ", denoted by " Neut$\mathrm{A}^{\prime}$.and from on, neutrosophy became the basis of neutrosophic set theory, neutrosophic logic, neutrosophic probability, and neutrosophic statistics . In 2012 neutrosophic crisp sets have been investigated by Salma et,al [8], [9], [10], [11], [12], [13].

On the other hand, scientists have started to color most domains of classical mathematics such as: topology[14], algebraic structures, relation theory, and differential measure theory .etc. In mathematics, topology (from the Greek, place and study) is concerned with the properties of space that are preserved under continuous deformations, such as stretching, crumpling and bending, but not tearing or gluing.Fuzzy logic [15],[16] has proved to be a powerful tool for decisionmaking systems, such as expert systems. Fuzzy set theory has already been used in the expert systems. Sanchez [17] formulated the diagnostic models involving fuzzy matrices representing the knowledge. Esogbue and Elder utilized fuzzy analysis to the model. Meenakshi and Kaliraja [18] have extended Sanchez's approach for any application using the representation of an interval valued fuzzy matrix. They have also introduced the arithmetic mean matrix of an interval valued fuzzy matrix and directly applied Sanchez's method of the application on it. Fuzzy set theory also plays an important role in the Decision Making. Decision Making is a most important scientific, social and economic endeavor. In classical crisp decision making theories, decisions are made under conditions of certainty but in real life situations this is not possible which gives rise to fuzzy decision making theories. For decision making in fuzzy environment one may refer Bellman and Zadeh [19],[20] .

In this paper, we would like to discuss how fuzzy set theory [21],[22] and fuzzy logic [23] can be used for developing knowledge based systems using triangular fuzzy number matrices by converting the information system to fuzzy matrices to take the suitable decision making and take the decision with degree.

Definition 1.1 [24] (Triangular fuzzy number matrix) Triangular fuzzy number matrix of order $m \times n$ is defined as $\mathrm{A}=\left(\mathrm{a}_{\mathrm{ij}}\right)_{m \times n}$ where $\mathrm{a}_{\mathrm{ij}}=\left(\mathrm{a}_{\mathrm{ijL}}, \mathrm{a}_{\mathrm{ijM}}, \mathrm{a}_{\mathrm{iju}}\right)$ is the $i j^{\text {th }}$ element of $\mathrm{A} \cdot \mathrm{a}_{\mathrm{ijL}}, \mathrm{a}_{\mathrm{ijU}}$ are the left and right spreads of $\mathrm{a}_{\mathrm{ij}}$ respectively and $\mathrm{a}_{\mathrm{ijM}}$ is the mean value.

Definition 1.2 (Maximum operation on triangular fuzzy number)

Let $\mathrm{A}=\left(\mathrm{a}_{\mathrm{ij}}\right)_{m \times n}$ where $\mathbf{a}_{\mathrm{ij}}=\left(\mathrm{a}_{\mathrm{ijL}}, \mathrm{a}_{\mathrm{ijM}}, \mathrm{a}_{\mathrm{ijU}}\right)$ and $\mathbf{B}=\left(\mathbf{b}_{\mathrm{ij}}\right)_{m \times n}$ whereby

$b_{i j}=\left(b_{i j L}, b_{i j M}, b_{i j \mathrm{U}}\right)$ be two triangular fuzzy number matrices of same order. Then the maximum operation on it is given by: $\mathbf{L}_{\max }=\max (\mathrm{A}, \mathrm{B})=\left(\sup \left\{\mathbf{a}_{i j} ; \mathbf{b}_{i j}\right\}\right)$, where:

$\sup \left\{\mathrm{a}_{i j}, \mathrm{~b}_{i j}\right\}=\left(\sup \left(\mathrm{a}_{i j L} ; \mathrm{b}_{i j L}\right), \sup \left(\mathrm{a}_{i j M} ; \mathrm{b}_{i j M}\right), \sup \left(\mathrm{a}_{i j U} ; \mathrm{b}_{i j U}\right)\right)$ is the ${ }_{i j^{t h}}$ element of $\max (\mathrm{A}, \mathrm{B})$.

Definition 1.3 (Arithmetic mean (AM) for triangular fuzzy number)

Let $A=\left(\mathbf{a}_{1}, \mathbf{a}_{2}, \mathbf{a}_{3}\right)$ be a triangular fuzzy number then,

$(A M)=\left(a_{1}+a_{2}+a_{3}\right) / 3$. The same condition holds for triangular fuzzy membership number.

\section{Methodology of Suitable Decision}

To find suitable decision for set of objects corresponding set of fuzzy parameters the values of membership function $\mu_{a_{i j}}(x)$ must satisfy $0 \leq \mu_{a_{i j}}(x) \leq 1$ and computable with formulated measure on the parameters. So, we can put the 
procedure of obtained suitable decision as follows:

Step 1: Construct the triangle fuzzy matrices.

Step 2: Convert the elements of triangle fuzzy number matrix into its membership function

$$
\mu_{a_{i j}}=\left(\frac{a_{i j L}}{100} \leq \frac{a_{i j M}}{100} \leq \frac{a_{i j U}}{100}\right) .
$$

Step 3: Compute the relation matrices [18] which help the decision maker to strongly confirm the correct decision.

Step 4: Now, we can take the suitable decision with degree after step 3 .

\section{APPLICATION OF FM IN SELECTING SPECIALIZATION}

In Egypt, Faculty of Engineering, Tanta University, we suffer from lack of specialization and how and why choosing the department to each student where the famous in Egypt each student chooses the department according to that its famous and ignoring that if this student is eligible to this department or not [25]. So, we need to choose the suitable department to each student by its degree of each object where each department needs to be excellent in specific objects.

\section{Example 1.1}

- In electrical department need the student to be excellent in Mathematical, Physics and Computer (Logic).

- In Architecture department need the student to be excellent in drawing engineering and Computer (Logic).

- In Mechanics department need the student to be excellent in drawing engineering, mechanics and Computer (Logic).

- In civil department need the student to be excellent in drawing engineering and Computer (Logic).

Let $S=\left\{S_{1}, S_{2}, S_{3}, S_{4}\right\}$ be the set of students, $D=$ (architecture, electrical, civil, mechanics\} be the set of department and $S u=$ (logic, Mathematics, drawing engineering, Physics, mechanics $\}$ be the set of subjects related to the departments. We assume the above students sit for examinations (i.e. over 10 marks total) on the above mentioned subjects to determine their department placements and choices. After the various examinations, the students obtained the following marks (see Table I). The departments and related subjects' requirements (see Table II).

TABLE I

STUDENTS VS SUBJECTS

\begin{tabular}{|l|c|r|r|r|r|}
\hline & Logic & Mathematics & $\begin{array}{l}\text { Drawing } \\
\text { engineeri }\end{array}$ & Physics & mechanics \\
\hline$S_{1}$ & $(8,9,10)$ & $(8,9,10)$ & $(5,6,7)$ & $(8,9,10)$ & $(4,5,6)$ \\
\hline$S_{2}$ & $(4,5,6)$ & $(5,6,7)$ & $(4,5,6)$ & $(3,4,5)$ & $(6,7,8)$ \\
\hline$S_{3}$ & $(6,7,8)$ & $(5,6,7)$ & $(6,7,8)$ & $(4,5,6)$ & $(3,4,5)$ \\
\hline$S_{4}$ & $(5,6,7)$ & $(7,8,9)$ & $(5,6,7)$ & $(5,6,7)$ & $(4,5,6)$ \\
\hline
\end{tabular}

TABLE II

DEPARTMENTS VS SUBJECTS

\begin{tabular}{|c|c|c|c|c|}
\hline & Architecture & Electrical & \multicolumn{1}{l|}{ Civil } & Mechanics \\
\hline Logic & $(7,8,9)$ & $(7,8,9)$ & $(7,8,9)$ & $(5,6,7)$ \\
\hline Mathematics & $(4,5,6)$ & $(8,9,10)$ & $(6,7,8)$ & $(7,8,9)$ \\
\hline $\begin{array}{c}\text { Drawing } \\
\text { engineering }\end{array}$ & $(8,9,10)$ & $(4,5,6)$ & $(8,9,10)$ & $(7,8,9)$ \\
\hline Physics & $(4,5,6)$ & $(7,8,9)$ & $(4,5,6)$ & $(4,5,6)$ \\
\hline Mechanics & $(4,5,6)$ & $(4,5,6)$ & $(5,6,7)$ & $(8,9,10)$ \\
\hline
\end{tabular}

\section{Step 1:}

By converting the information system to the triangular fuzzy number matrix $\left(F_{1}, S\right)$ is another parameterized family of triangular fuzzy number matrix and gives a collection of approximate description of the student-subject in the university. Thus the triangular fuzzy number matrix $\left(F_{1}, S\right)$ represents a relation matrix $M_{S S U}$ called studentsubject matrix given by:

$$
\begin{aligned}
& F_{1}\left(S U_{1}\right)=\left[\begin{array}{l}
<S_{1},(8,9,10)>;<S_{2},(4,5,6)>; \\
<S_{3}(6,7,8)>;<S_{4},(5,6,7)>
\end{array}\right] \\
& F_{1}\left(S U_{2}\right)=\left[\begin{array}{l}
<S_{1},(8,9,10)>;<S_{2},(5,6,7)>; \\
<S_{3}(5,6,7)>;<S_{4}(7,8,9)>
\end{array}\right] \\
& F_{1}\left(S_{3}\right)=\left[\begin{array}{l}
<S_{1},(5,6,7)>;<S_{2}(4,5,6)>; \\
<S_{3},(6,7,8)>;<S_{4},(5,6,7)>
\end{array}\right] \\
& F_{1}\left(S_{4}\right)=\left[\begin{array}{l}
<S_{1},(8,9,10)>;<S_{2}(3,4,5)>; \\
<S_{3}(4,5,6)>;<S_{4}(5,6,7)>
\end{array}\right] \\
& F_{1}\left(S U_{5}\right)=\left[\begin{array}{l}
<S_{1}(4,5,6)>;<S_{2}(6,7,8)>; \\
<S_{3}(3,4,5)>;<S_{4}(4,5,6)>
\end{array}\right] \\
& \begin{array}{lllll}
S U_{1} & S U_{2} & S U_{3} & S U_{4} & S U_{5}
\end{array} \\
& M_{S S U}=\frac{\mathrm{S}_{1}}{\mathrm{~S}_{3}}\left(\begin{array}{ccccc}
(8,9,10) & (8,9,10) & (5,6,7) & (8,9,10) & (4,5,6) \\
& \mathrm{S}_{4}
\end{array}\left(\begin{array}{lllll}
(4,5,6) & (5,6,7) & (4,5,6) & (3,4,5) & (6,7,8) \\
(5,7,8) & (5,6,7) & (6,7,8) & (4,5,6) & (3,4,5) \\
(5,6) & (7,8,9) & (5,6,7) & (5,6,7) & (4,5,6)
\end{array}\right)\right.
\end{aligned}
$$

By converting the information system to the triangular fuzzy number matrix (F,D) is another parameterized family of triangular fuzzy number matrix and gives a collection of approximate description of the departmentsubject in the university. Thus the triangular fuzzy number matrix (F, D) represents a relation matrix $M_{D S U}$ called department-subject matrix given by:

$$
\begin{aligned}
& \mathrm{F}\left(\mathrm{D}_{1}\right)=\left[\begin{array}{l}
<\mathrm{SU}_{1},(7,8,9)>;<\mathrm{SU}_{2},(4,5,6)>;<\mathrm{SU}_{3}(8,9,10)>; \\
<\mathrm{SU}_{4},(4,5,6)>;<\mathrm{SU}_{5},(4,5,6)>
\end{array}\right] \\
& \mathrm{F}\left(\mathrm{D}_{2}\right)=\left[\begin{array}{l}
<\mathrm{SU}_{1},(7,8,9)>;<\mathrm{SU}_{2},(8,9,10)>;<\mathrm{SU}_{3},(4,5,6)>; \\
<\mathrm{SU}_{4},(7,8,9)>;<\mathrm{SU}_{5},(4,5,6)>
\end{array}\right] \\
& \mathrm{F}\left(\mathrm{D}_{3}\right)=\left[\begin{array}{l}
<\mathrm{SU}_{1},(7,8,9)>;<\mathrm{SU}_{2},(6,7,8)>;<\mathrm{SU}_{3}(8,9,10)>; \\
<\mathrm{SU}_{4},(4,5,6)>;<\mathrm{SU}_{5},(5,6,7)>
\end{array}\right]
\end{aligned}
$$




$$
\begin{aligned}
& \mathrm{F}\left(\mathrm{D}_{4}\right)=\left[\begin{array}{l}
<\mathrm{SU}_{1},(5,6,7)>;<\mathrm{SU}_{2},(7,8,9)>;<\mathrm{SU}_{3}(7,8,9)>; \\
<\mathrm{SU}_{4},(4,5,6)>;<\mathrm{SU}_{5},(8,9,10)>
\end{array}\right] \\
& \begin{array}{cccc}
D_{1} & D_{2} & D_{3} & D_{4}
\end{array}
\end{aligned}
$$

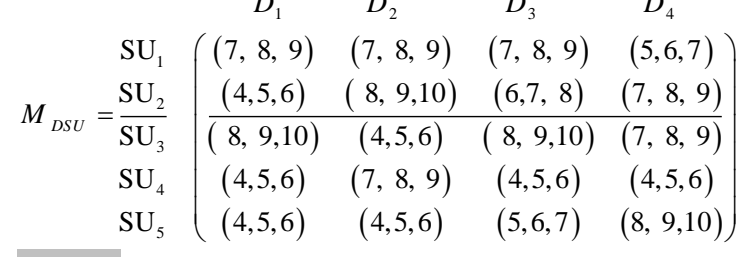

\section{Step 2:}

$$
\begin{aligned}
& \left(\mathrm{M}_{S S U}\right)_{\text {men }}= \\
& \begin{array}{lllll}
S U_{1} & S U_{2} & S U_{3} & S U_{4} & S U_{5}
\end{array} \\
& \mathrm{~S}_{1} \quad\left(\begin{array}{lllll}
(0.8,0.9,1) & (0.8,0.9,1) & (0.5,0.6,0.7) & (0.8,0.9,1) & (0.4,0.5,0.6)
\end{array}\right) \\
& \begin{array}{l|llllll}
S_{2} & (0.4,0.5,0.6) & (0.5,0.6,0.7) & (0.4,0.5,0.6) & (0.3,0.4,0.5) & (0.6,0.7,0.8) \\
\hline
\end{array} \\
& \begin{array}{llllll}
S_{3} & (0.6,0.7,0.8) & (0.5,0.6,0.7) & (0.6,0.7,0.8) & (0.4,0.5,0.6) & (0.3,0.4,0.5)
\end{array} \\
& \begin{array}{llllll}
S_{4} & (0.5,0.6,0.7) & (0.7,0.8,0.9) & (0.5,0.6,0.7) & (0.5,0.6,0.7) & (0.4,0.5,0.6)
\end{array}
\end{aligned}
$$

$$
\begin{aligned}
& \left(\mathrm{M}_{D S U}\right)_{m e n}= \\
& \begin{array}{cccc}
D_{1} & D_{2} & D_{3} & D_{4}
\end{array} \\
& \begin{array}{lllll}
\mathrm{SU}_{1} \quad(0.7,0.8,0.9) & (0.7,0.8,0.9) & (0.7,0.8,0.9) & (0.5,0.6,0.7)
\end{array} \\
& \begin{array}{l|llll}
\mathrm{SU}_{2} & (0.4,0.5,0.6) & (0.8,0.9,1) & (0.6,0.7,0.8) & (0.7,0.8,0.9) \\
\hline & (0.8,0.9,1) & (0.4,0.5,0.6) & (0.8,0.9,1) & (0.7,0.8,0.9)
\end{array} \\
& \begin{array}{lllll}
\mathrm{SU}_{3} & (0.8,0.9,1) & (0.4,0.5,0.6) & (0.8,0.9,1) & (0.7,0.8,0.9)
\end{array} \\
& \begin{array}{l|llll}
\mathrm{SU}_{4} & (0.4,0.5,0.6) & (0.7,0.8,0.9) & (0.4,0.5,0.6) & (0.4,0.5,0.6)
\end{array} \\
& \left.\begin{array}{lllll}
\mathrm{SU}_{5} & (0.4,0.5,0.6) & (0.4,0.5,0.6) & (0.5,0.6,0.7) & (0.8,0.9,1)
\end{array}\right)
\end{aligned}
$$

\begin{tabular}{|c|c|c|c|c|}
\hline$S_{1}$ & $((0.1,0.3,0.5)$ & $(0.2,-0.5,0.6)$ & $(0.1,0.3,0.5)$ & $(0.1,0.3,0.5)$ \\
\hline & $(-0.2,0,0)$ & $(-0.1,0.1,0.2)$ & $(-0.1,0.1,0.2)$ & $(0,0.2,0.3)$ \\
\hline & $(0.1,0.2,0.3)$ & $(0,0.2,0.4)$ & $(0.1,0.1,0.3)$ & $(-0.1,0.1,0.3)$ \\
\hline & $(-0.1,0.1,0.3)$ & $(0.2,0.3,0.5)$ & $(0.1,0.2,0.4)$ & $(0.1,0.3,0.5)$ \\
\hline
\end{tabular}

\begin{tabular}{|c|c|c|c|c|}
\hline & $D_{1}$ & $D_{2}$ & $D_{3}$ & $D_{4}$ \\
\hline $\mathrm{S}_{1}$ & 0.03 & 0.1 & 0.03 & 0.03 \\
\hline$M_{6}=S_{2}$ & -0.07 & 0.07 & 0.07 & 0.17 \\
\hline $\mathrm{S}_{3}$ & 0.21 & 0.2 & 0.17 & 0.1 \\
\hline $\mathrm{S}_{4}$ & 0.1 & 0.23 & 0.33 & 0.3 \\
\hline
\end{tabular}

\section{Step 3:}

Computing the following relation matrices

$$
\left.\begin{array}{lcccc}
\multicolumn{5}{c}{\mathrm{M}_{1}=\left(\mathrm{M}_{S S U}\right)_{\text {men }}(.)\left(\mathrm{M}_{D S U}\right)_{\text {men }}=} \\
D_{1} & D_{2} & D_{3} & D_{4} \\
\mathrm{~S}_{1} & (0.7,0.8,0.9) & (0.8,0.9,1) & (0.7,0.8,0.9) & (0.7,0.8,0.9) \\
\mathrm{S}_{2} & (0.4,0.5,0.6) & (0.5,0.6,0.7) & (0.5,0.6,0.7) & (0.6,0.7,0.8) \\
\mathrm{S}_{3} & (0.6,0.7,0.8) & (0.6,0.7,0.8) & (0.6,0.7,0.8) & (0.6,0.7,0.8) \\
\mathrm{S}_{4} & (0.5,0.6,0.7) & (0.7,0.8,0.9) & (0.6,0.7,0.8) & (0.7,0.8,0.9)
\end{array}\right)
$$

$$
\begin{aligned}
& \mathrm{M}_{2}=\left(\mathrm{M}_{S S U}\right)_{m e n}(.)\left(\mathrm{J}(-)\left(\mathrm{M}_{D S U}\right)_{m e n}\right)= \\
& \begin{array}{cccc}
D_{1} & D_{2} & D_{3} & D_{4}
\end{array} \\
& S_{1} \quad\left(\begin{array}{llll}
(0.6,0.5,0.4) & (0.6,0.5,0.4) & (0.6,0.5,0.4) & (0.6,0.5,0.4)
\end{array}\right. \\
& \mathrm{S}_{2} \quad\left(\begin{array}{lllll}
(0.6,0.5,0.4) & (0.6,0.5,0.4) & (0.5,0.4,0.4) & (0.4,0.4,0.5)
\end{array}\right. \\
& \begin{array}{llllll}
\mathrm{S}_{3} & (0.5,0.5,0.4) & (0.6,0.5,0.4) & (0.4,0.5,0.4) & (0.5,0.5,0.4)
\end{array} \\
& \begin{array}{lllll}
\mathrm{S}_{4} & (0.6,0.5,0.4) & (0.5,0.5,0.4) & (0.5,0.5,0.4) & (0.5,0.5,0.4)
\end{array}
\end{aligned}
$$

$$
\begin{aligned}
& \mathrm{M}_{3}=\left(\mathrm{J}(-)\left(\mathrm{M}_{S S U}\right)_{m e n}\right)(.)\left(\mathrm{M}_{D S U}\right)_{m e n}= \\
& \begin{array}{ccccc}
D_{1} & D_{2} & D_{3} & D_{4}
\end{array} \\
& \mathrm{~S}_{1} \quad\left(\begin{array}{llll}
(0.5,0.5,0.4) & (0.4,0.5,0.4) & (0.5,0.5,0.4) & (0.5,0.5,0.4)
\end{array}\right) \\
& \mathrm{S}_{2} \quad\left(\begin{array}{lllll}
0.6,0.5,0.6) & (0.6,0.5,0.5) & (0.6,0.5,0.5) & (0.6,0.5,0.4)
\end{array}\right. \\
& \begin{array}{llllll}
\mathrm{S}_{3} & (0.4,0.5,0.5) & (0.6,0.5,0.4) & (0.5,0.6,0.5) & (0.7,0.6,0.5)
\end{array} \\
& \mathrm{S}_{4} \quad\left(\begin{array}{lllll}
(0.5,0.5,0.4) & (0.5,0.5,0.4) & (0.5,0.5,0.4) & (0.6,0.5,0.4)
\end{array}\right)
\end{aligned}
$$

$$
\left.\begin{array}{rcccc} 
& D_{1} & D_{2} & D_{3} & D_{4} \\
\mathrm{~S}_{1} & (0.6,0.5,0.4) & (0.6,0.5,0.4) & (0.6,0.5,0.4) & (0.6,0.5,0.4) \\
\mathrm{M}_{4}=\max \left\{\mathrm{M}_{2}, \mathrm{M}_{3}\right\}=\mathrm{S}_{2} & (0.6,0.5,0.6) & (0.6,0.5,0.5) & (0.6,0.5,0.5) & (0.6,0.5,0.5) \\
\mathrm{S}_{3} & (0.5,0.5,0.5) & (0.6,0.5,0.4) & (0.5,0.6,0.5) & (0.7,0.6,0.5) \\
\mathrm{S}_{4} & (0.6,0.5,0.4) & (0.5,0.5,0.4) & (0.5,0.5,0.4) & (0.6,0.5,0.4)
\end{array}\right)
$$

$$
\mathrm{M}_{5}=\mathrm{M}_{1}(-) \mathrm{M}_{4}=
$$$$
\begin{array}{ccccc}
D_{1} & D_{2} & D_{3} & D_{4}
\end{array}
$$

Step 4:

$\mathrm{S}_{1}$ can select electrical (Electrical Engineer), mechanics (Mechanical Engineer) or architecture (architecture Engineer) and by the requesting department or the biggest degree chosen this student in the suitable department. $S_{2}$ is to read mechanics (Mechanical Engineer), $S_{3}$ is to read architecture (architecture Engineer) or civil (civil Engineer) and by the requesting department or the biggest degree chosen this student in the suitable department. And $S_{4}$ is to read civil (civil Engineer). So, we deduce that this case study presented in this mark can be applied in many real life applications. For example: political or social case.

\section{ROUGH SET CONCEPTS ON SELECTING SPECIALIZATION}

In this paper, we connect the Rough set theory [26] with Horizontal Curve Design by classifying the various roots by its diameters and make decision for suitable velocity.

Definition 1.4: Let $A$ be a fuzzy set and $\mu_{A}(x)$ is membership for $x \in A$ then

$A^{\alpha}=\{x: \mu(\alpha) \geq \alpha\}, S_{\alpha}=\left\{A^{\alpha},\left(A^{\alpha}\right)^{c}\right\}, 0 \leq \alpha \leq 1$.

Definition 1.5: Let $A$ and $B$ be fuzzy sets and $R$ is relation from $\boldsymbol{A} \times \boldsymbol{B}$ to $\stackrel{B}{B}$ where $\boldsymbol{B}$ is crisp set. So, 
$\boldsymbol{R}_{A}=\left\{\left\{x_{i}\right\}:\left(\left(x_{i}, y_{i}\right), \mu\left(y_{i}\right)\right) \in R\right.$ such that $\mu\left(y_{i}\right) \geq \alpha$ Example 1.3

, $S_{\alpha}^{A}=\left\{R_{A},\left(R_{A}\right)^{c}\right\}$.Example 3.1: If $\alpha \geq 0.8$ be

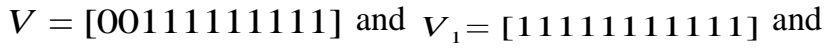
when $\quad \alpha \geq 0.9$ be $V=[00000011111]$ and $V_{1}=[00001111111]$, as given by (see Table III).

Using the above definitions, we obtain the following approximations:

Using the above definitions, we obtain the following approximations: Case 1: At $\alpha \geq 0.8$, The comparison lower approximation $L_{V_{1}}^{1}(X)=\phi$, the comparison upper approximation

$U_{V_{1}}^{1}(X)=\left\{R_{1}, R_{2}, R_{3}, R_{4}, R_{5}, R_{6}, R_{7}, R_{8}, R_{9}, R_{10}, R_{11}\right\}$ $S_{0.8}^{V_{1}}=\left\{\left\{R_{1}, R_{2}\right\},\left\{R_{3}, R_{4}, R_{5}, R_{6}, R_{7}, R_{8}, R_{9}, R_{10}, R_{11}\right\}\right\}$ The decision lower approximation $L_{V}^{2}(X)=\left\{R_{1}, R_{2}\right\}$ and $S_{0.8}^{V}=\left\{R_{1}, R_{2}, R_{3}, R_{4}, R_{5}, R_{6}, R_{7}, R_{8}, R_{9}, R_{10}, R_{11}\right\}$ the decision upper approximation

$S_{0.9}^{V_{1}}=\left\{\left\{R_{1}, R_{2}, R_{3}, R_{4}, R_{5}, R_{6},\right\},\left\{R_{7}, R_{8}, R_{9}, R_{10}, R_{11}\right\}\right\}$ $S_{0.9}^{V}=\left\{\left\{R_{1}, R_{2}, R_{3}, R_{4}\right\},\left\{R_{5}, R_{6}, R_{7}, R_{8}, R_{9}, R_{10}, R_{11}\right\}\right\}$

Definition 1.6: Let $A, B, C$ be fuzzy sets and $\mu_{A}(x), \mu_{B}(y), \mu_{C}(z)$ be a membership of $x \in A, y \in B, z \in C$ then the $\alpha-$ level decision classification of $A$ can be defined as:

$D_{\alpha}=\left\{\left\{x_{i}\right\}: x_{i} \in A,\left(x_{i}, y_{i}, z_{i}\right) \in A \times B \times C\right.$ and $\left.\mu_{B}\left(y_{i}\right) \geq \mu_{C}\left(z_{i}\right)\right\}$ $D^{A}=\left\{D_{A},\left(D_{A}\right)^{c}\right\}$

\section{Example 1.2}

Let $X=\left\{x: V_{1} \geq V=\right.$ Yes $\}$, as given by Table III. In fact, the set $X$ consists of three objects: $\boldsymbol{R}_{1}, \boldsymbol{R}_{2}, \boldsymbol{R}_{3}, \boldsymbol{R}_{4}, \boldsymbol{R}_{5}, \boldsymbol{R}_{6}, \boldsymbol{R}_{7}, \boldsymbol{R}_{8}$. Now, we want to describe this set in terms of the set of conditional attributes $A=\{$ suitable or not $\}$.

\section{NEW APPROXIMATION OPERATION ON HORIZONTAL CURVE DESIGN}

In this paper, we also used lower and upper approximations of above set are important to reduce the number of sets to make suitable decision. If we used uncertainty concepts to determine any set, we must find two sets one of them included and other contained in this set. Under classification of set of parameter and condition, we formed four approximations operators as follows: Definition 1.7: Let $A_{1}, A_{2}, \ldots, A_{n}$ be fuzzy sets of comparison values with set $A$ then comparison lower approximation of set $A_{i}$ can be defined as: $L_{A_{i}}^{1}(X)=\left\{k: k \in S_{\alpha}^{A_{i}}\right.$ and $\left.k \subseteq X\right\}$ and comparison upper approximation of set $A_{i}$ can be defined as: $U_{A_{i}}^{1}(X)=\left\{k: k \in S_{\alpha}^{A_{i}}\right.$ and $\left.X \cap k \neq \phi\right\} \quad$ where $X \subseteq U, U$ is universal set.

Definition 1.8: Let $A_{1}, A_{2}, \ldots, A_{n}$ be fuzzy sets of comparison values with set $A$ then decision lower approximation of $\boldsymbol{X} \subseteq \boldsymbol{U}, \boldsymbol{U}$ is universal set can be defined as: $L_{A}^{2}(X)=\left\{k: k \in D_{\alpha}^{A}\right.$ and $\left.k \subseteq X\right\}$ and decision upper approximation of $\boldsymbol{X} \subseteq \boldsymbol{U}, \boldsymbol{U}$ is universal set can be defined as:

$U_{A}^{2}(X)=\left\{k: k \in D_{\alpha}^{A}\right.$ and $\left.X \cap k \neq \phi\right\}$
$U_{V}^{2}(X)=\left\{R_{1}, R_{2}, R_{3}, R_{4}, R_{5}, R_{6}, R_{7}, R_{8}, R_{9}, R_{10}, R_{11}\right\}$

Case 2: At $\alpha \geq 0.9$, The comparison lower approximation $L_{V_{1}}^{1}(X)=\left\{R_{1}, R_{2}, R_{3}, R_{4}\right\}$, the comparison upper approximation

$U_{V_{1}}^{1}(X)=\left\{R_{1}, R_{2}, R_{3}, R_{4}, R_{5}, R_{6}, R_{7}, R_{8}, R_{9}, R_{10}, R_{11}\right\}$ the decision lower approximation

$\boldsymbol{L}_{V}^{2}(X)=\left\{\boldsymbol{R}_{1}, \boldsymbol{R}_{2}, \boldsymbol{R}_{3}, \boldsymbol{R}_{4}, \boldsymbol{R}_{5}, \boldsymbol{R}_{6}\right\}$ and the decision upper approximation

$U_{V}^{2}(X)=\left\{R_{1}, R_{2}, R_{3}, R_{4}, R_{5}, R_{6}, R_{7}, R_{8}, R_{9}, R_{10}, R_{11}\right\}$

One can easily the following properties of approximations. Proposition 1.1 Let $L_{V_{1}}^{1}(X)$ and $L_{V}^{2}(X)$ be comparison and decision lower approximation then the following are satisfied.

i. $L_{V_{1}}^{1}(\phi)=\phi$.

\section{Proof:}

Let $L_{V_{1}}^{1}(X)=\phi$, If $X=\phi$, then $L_{V_{1}}^{1}(\phi)=\phi$.

By the same way, $L_{V}^{2}(\phi)=\phi$.

ii. $L_{V_{1}}^{1}(U)=U$.

Proof:

Let $L_{V_{1}}^{1}(X)=U$, If $X=U$, then $L_{V_{1}}^{1}(U)=U$.

By the same way, $L_{V}^{2}(U)=U$.

iii. $L_{V_{1}}^{1}(X \cap Y)=L_{V_{1}}^{1}(X) \cap L_{V_{1}}^{1}(Y)$.

Proof:

Let $x \in L_{V_{1}}^{1}(X) \cap L_{V_{1}}^{1}(Y) \Rightarrow x \in L_{V_{1}}^{1}(X) \Rightarrow \exists k \in S_{\alpha}^{A_{i}}$ and $k \subseteq X$;

$x \in L_{V_{1}}^{1}(Y) \Rightarrow \exists k \in S_{\alpha}^{A_{i}}$ and $k \subseteq Y$, then $k \subseteq X \cap Y$ from Defination 3.1 then $k \subseteq L_{V_{1}}^{1}(X \cap Y)$, then $L_{V_{1}}^{1}(X \cap Y)=L_{V_{1}}^{1}(X) \cap L_{V_{1}}^{1}(Y)$.

By the same way, $L_{V}^{2}(X \cap Y)=L_{V}^{2}(X) \cap L_{V}^{2}(Y)$. iv. $X \subseteq Y$ implies $L_{V_{1}}^{1}(X) \subseteq L_{V_{1}}^{1}(Y)$.

\section{Proof:}

If $X \subseteq Y$, then $x \in X \Rightarrow x \in L_{V_{1}}^{1}(X) \Rightarrow x \in L_{V_{1}}^{1}(Y) \Rightarrow L_{V_{1}}^{1}(X) \subseteq L_{V_{1}}^{1}(Y)$ By the same way, $X \subseteq Y$ implies $L_{V}^{2}(X) \subseteq L_{V}^{2}(Y)$.

v. $L_{V_{1}}^{1}(X \cup Y) \supseteq L_{V_{1}}^{1}(X) \cup L_{V_{1}}^{1}(Y)$.

Proof:

If

$x \in X$ or $x \in Y \Rightarrow x \in L_{V_{1}}^{1}(X)$ or $x \in L_{V_{1}}^{1}(Y) \Rightarrow x \in L_{V_{1}}^{1}(X) \cup x \in L_{V_{1}}^{1}(Y) \subseteq L_{V_{1}}^{1}(X \cup Y)$. 
Thus $x \in L_{V_{1}}^{1}(X \cup Y)$. It is easy to notice that the reverse implication is not true in general,

for $L_{V_{1}}^{1}(X \cup Y) \neq L_{V_{1}}^{1}(X) \cup L_{V_{1}}^{1}(Y)$.

By the same way, $L_{V}^{2}(X \cup Y) \supseteq L_{V}^{2}(X) \cup L_{V}^{2}(Y)$.

Proposition 1.2 Let $U_{V_{1}}^{1}(X)$ and $U_{V}^{2}(X)$ be comparison and decision upper approximation then the following are satisfied.

i. $U_{V_{1}}^{1}(\phi)=\phi$.

ii. $U_{V_{1}}^{1}(U)=U$.

iii. $U_{V_{1}}^{1}(X \cup Y)=U_{V_{1}}^{1}(X) \cup U_{V_{1}}^{1}(Y)$.

iv. $X \subseteq Y$ implies $U_{V_{1}}^{1}(X) \subseteq U_{V_{1}}^{1}(Y)$.

v. $U_{V_{1}}^{1}(X \cap Y) \subseteq U_{V_{1}}^{1}(X) \cap U_{V_{1}}^{1}(Y)$.

Proposition 1.3 Let $L_{V_{1}}^{1}(X)$ and $L_{V}^{2}(X)$ be comparison and decision lower approximation, $U_{V_{1}}^{1}(X)$ and $U_{V}^{2}(X)$ be comparison and decision upper approximation then the following are satisfied.

i. $L_{V_{1}}^{1}(X) \subseteq X \subseteq U_{V_{1}}^{1}(X)$.

Proof:

If $\quad x \in X \Rightarrow x \in L_{V_{1}}^{1}(X)$ and $x \in U_{V_{1}}^{1}(X)$

But $L_{V_{1}}^{1}(X) \subseteq X$ and $X \subseteq U_{V_{1}}^{1}(X)$

, then $L_{V_{1}}^{1}(X) \subseteq X \subseteq U_{V_{1}}^{1}(X)$.

By the same way, $L_{V}^{2}(X) \subseteq X \subseteq U_{V}^{2}(X)$.

ii. $L_{V_{1}}^{1}(U-X)=U-U_{V_{1}}^{1}(X)$.

Proof:

If $x \in(U-X) \Leftrightarrow x \in L_{V_{1}}^{1}(U-X)$

So

$x \in(U-X) \Leftrightarrow x \in U-U_{V_{1}}^{1}(X) \Leftrightarrow x \notin U_{V_{1}}^{1}(X) \Leftrightarrow$ non $\mathrm{x} \in U_{V_{1}}^{1}(X)$

, then $L_{V_{1}}^{1}(U-X)=U-U_{V_{1}}^{1}(X)$.

By the same way, $L_{V}^{2}(U-X)=U-U_{V}^{2}(X)$.

iii. $U_{V_{1}}^{1}(U-X)=U-L_{V_{1}}^{1}(X)$.

Proof:

If $x \in(U-X) \Leftrightarrow x \in U_{V_{1}}^{1}(U-X)$

So

$x \in(U-X) \Leftrightarrow x \in U-L_{V_{1}}^{1}(X) \Leftrightarrow x \notin L_{V_{1}}^{1}(X) \Leftrightarrow$ non $\mathrm{x} \in L_{V_{1}}^{1}(X)$

, then $U_{V_{1}}^{1}(U-X)=U-L_{V_{1}}^{1}(X)$.

By the same way, $U_{V}^{2}(U-X)=U-L_{V}^{2}(X)$.

iv. $L_{V_{1}}^{1}\left(L_{V_{1}}^{1}(X)\right)=U_{V_{1}}^{1}\left(L_{V_{1}}^{1}(X)\right)=L_{V_{1}}^{1}(X)$.

Proof:

Let $L_{V_{1}}^{1}(X)=X$ and $U_{V_{1}}^{1}(X)=X$, If $X=L_{V_{1}}^{1}(X)$

, then $L_{V_{1}}^{1}\left(L_{V_{1}}^{1}(X)\right)=U_{V_{1}}^{1}\left(L_{V_{1}}^{1}(X)\right)=L_{V_{1}}^{1}(X)$.

By the same

way, $L_{V}^{2}\left(L_{V}^{2}(X)\right)=U_{V}^{2}\left(L_{V}^{2}(X)\right)=L_{V}^{2}(X)$.

v. $U_{V_{1}}^{1}\left(U_{V_{1}}^{1}(X)\right)=L_{V_{1}}^{1}\left(U_{V_{1}}^{1}(X)\right)=U_{V_{1}}^{1}(X)$.

Proof:
Let $L_{V_{1}}^{1}(X)=X$ and $U_{V_{1}}^{1}(X)=X$, If $X=U_{V_{1}}^{1}(X)$

then $L_{V_{1}}^{1}\left(U_{V_{1}}^{1}(X)\right)=U_{V_{1}}^{1}\left(U_{V_{1}}^{1}(X)\right)=U_{V_{1}}^{1}(X)$.

By the same

e way, $U_{V}^{2}\left(U_{V}^{2}(X)\right)=L_{V}^{2}\left(U_{V}^{2}(X)\right)=U_{V}^{2}(X)$.

Where $-X$ denotes $U-X$.

It is easily seen that the lower and the upper approximations of a set, are respectively, the interior and the closure of this set in the topology generated by the indiscernibility relation

Since any set can measure by upper and lower approximations, which depends on the method of classifications by parameters. So, it is important to introducedthe various kinds or Rough notations on sets.

Definition 1.8 Let $U=\left\{R_{1}, R_{2}, \ldots, R_{n}\right\}$ be set of objects and $V_{1}=\left\{V_{1}, V_{2}, \ldots, V_{m}\right\}$ be set of comparison fuzzy parameters, then the following four categories of vagueness defined as:

1. A set $X$ is roughly $V_{1}$-definable, iff $L_{V_{1}}^{1}(X) \neq \phi$ and $U_{V_{1}}^{1}(X) \neq U$,

2. A set $X$ is internally $V_{1}$-undefinable, iff $L_{V_{1}}^{1}(X)=\phi$ and $U_{V_{1}}^{1}(X) \neq U$,

3. A set $X$ is externally $V_{1}$-undefinable, iff $L_{V_{1}}^{1}(X) \neq \phi$ and $U_{V_{1}}^{1}(X)=U$,

4. A set $X$ is totally $V_{1}$-undefinable, iff $L_{V_{1}}^{1}(X)=\phi$ and $U_{V_{1}}^{1}(X)=U$

Definition 1.9 Let $U=\left\{R_{1}, R_{2}, \ldots, R_{n}\right\}$ be set of objects and $V=\left\{V_{1}, V_{2}, \ldots, V_{m}\right\}$ be a set of standard fuzzy parameters defined on $U$ the following four categories of vagueness defined as:

1. A set $X$ is roughly $V$-definable, iff $L_{V}^{2}(X) \neq \phi$ and $U_{V}^{2}(X) \neq U$

2. A set $X$ is internally $V$-undefinable, iff $L_{V}^{2}(X)=\phi$ and $U_{V}^{2}(X) \neq U$,

3. A set $X$ is externally $V$-undefinable, iff $L_{V}^{2}(X) \neq \phi$ and $U_{V}^{2}(X)=U$,

4. A set $X$ is totally $V$-undefinable, iff $L_{V}^{2}(X)=\phi$ and $U_{V}^{2}(X)=U$.

The intuitive meaning of this classification is the following:

- A set $X$ is roughly $V$-definable means that with the help of $A$ we are able to decide for some elements of $U$ that they belong to $X$ and for some elements of $U$ that they belong to $-X$.

- A set $X$ is internally $V$-undefinable means that using $A$ we are able to decide for some elements of $U$ that they belong to $-X$, but we are unable to decide for any element of $U$ whether it belong to $X$. 
- A set $X$ is externally $V$-undefinable means that using $A$ we are able to decide for some elements of $U$ that they belong to $X$, but we are unable to decide for any element of $U$ whether it belongs to $-X$.

- A set $X$ is totally $V$-undefinable means that using $A$ we are unable to decide for any element of $U$ whether it belongs to $X$ or $-X$.

A rough set can be also characterized numerically by the

- following coefficient:

$$
\begin{aligned}
& \alpha_{A}^{11}(X)=\frac{\left|L_{V_{1}}^{1}(X)\right|}{\left|U_{V_{1}}^{1}(X)\right|} \quad, \quad \alpha_{A}^{12}(X)=\frac{\left|L_{V_{1}}^{1}(X)\right|}{\left|U_{V}^{2}(X)\right|} \\
& \alpha_{A}^{21}(X)=\frac{\left|L_{V}^{2}(X)\right|}{\left|U_{V_{1}}^{1}(X)\right|}
\end{aligned}
$$

And by the same way, $\alpha_{A}^{22}(X)=\frac{\left|L_{V}^{2}(X)\right|}{\left|U_{V}^{2}(X)\right|}$ called the accuracy of approximation, where $X$ denotes the cardinally of $X \neq \phi$. Obviously $0 \leq \alpha_{A}(X) \leq 1$. If $\alpha_{A}(X)=1$, $X$ is crisp with respect to $A$ ( $X$ is precise with respect to $A$ ), and otherwise, if $\alpha_{A}(X)<1, X$ is rough with respect to $A$ ( $X$ is vague with respect to $A$ ).

\section{SUMMARY}

We review several concepts which will be used in this paper; the development from fuzzy set to its extensions for instance, the neutrosphic set, the intuitionistic set, the intuitionistic fuzzy set and display in this paper the fuzzy matrix that is quite interesting and useful in many application areas. We also display an application associate with it.

\section{CONCLUSION}

The case study presented in this work can be applied in many real life applications. For example: medicine, political or social cases.

\section{REFERENCES}

[1] G. Cantor, "On a property of the collection of all real algebraic numbers," Math., vol. 77, pp. 258-262, 1874.

[2] T. El., "On soft compact and soft pre-open sets, Annals of Fuzzy Mathematics and Informatics," vol. 17, no. 1, pp. 79-100, 2019.

[3] L. A. Zadeh, "Fuzzy sets," Information and control, vol. 8, no. 3, pp. 338-353, 1965.

[4] K. T. Atanassov, "Intuitionistic Fuzzy Sets. Proc. of Polish Symp. On Interval and Fuzzy mathematics," Poznan, pp. 23-26, 1983.

[5] K. T. Atanassov, "Intuitionistic fuzzy set," Fuzzy sets and Systems, vol. 20, no. 1, pp. 87-96, 1986.
[6] K. T. Atanassov, "More on Intuitionistic fuzzy sets," Fuzzy sets and Systems, vol. 33, pp. 37-46, 1989.

[7] K. T. Atanassov, "Operators Over Interval-valued Intuitionistic Fuzzy Sets," Fuzzy sets and Systems, vol. 64, pp. 159-174, 1994.

[8] A. A. Salama, "Neutrosophic crisp point \& Neutrosophic crisp ideals, Neutrosophic sets and Systems," vol. 1, no. 1, pp. 50-54, 2013.

[9] A. A. Salama, "The concept of neutrosophic set and basic properties of neutrosophic set operations " International University of Science, Engineering and Technology, 2012.

[10]S. A. A. A. A. Salama, "Generalized neutrosophic set and generalized neutrosophic spaces," Journal Computer Sci. Engineering, vol. 2, no. 7, pp. 129-132, 2012.

[11]S. A. B. A. A. Salama, F. Smarandache, "Neutrosophic crisp open set and neutrosophic crisp continuity via neutrosophic crisp ideals," I. J. Information Engineering and Electrical Business, vol. 3, pp. 1-8, 2014.

[12]F. S. A. A. Salama, "Neutrosophic crisp set theory," Education publisher Columbus, 2015.

[13]F. S. a. V. K. A. A. Salama, "Neutrosophic crisp sets \& Neutrosophic crisp topology spaces, Neutrosophic Sets and Systems," vol. 2, pp. 25-30, 2014.

[14]T. El, "Two types of separation axioms on supra soft topological spaces " Demonstratio Mathematica, vol. 52, no. 1, pp. 147-165, 2019.

[15]T. T. P. G. C. , " "Fuzzy Set, Fuzzy Logic, and Fuzzy Control Systems,"" CRC Press LLC, 2001.

[16]P. S. a. R. V. R. Sophia Porchelvi "An Application of Fuzzy Matrices In Medical Diagnosis," International Journal of Mathematical Analysis, vol. 9, no. 2, pp. 211216, 2015.

[17]E.Sanchez, "'Inverse of fuzzy relations, application to possibilty distribution and medical diagnosis," Fuzzy Sets and Systems," vol. 2, pp. 75-86, 1979.

[18]A. R. M. a. M.Kaliraja, ""An application of interval valued fuzzy matrices in medical diagnosis,"" International Journal of Mathematical Analysis, vol. 5, pp. 1791-1802, 2011.

[19]R. B. a. L.A.Zadeh, "Decision making in a fuzzy environment " Management Science," vol. 17, pp. 144164, 1970.

[20]G. J. K. , B. Yuan, "Fuzzy Sets and Fuzzy Logic: Theory and Applications," Prentice-Hall, International Inc., 1995

[21]Barnabas Bede, "Mathematics of Fuzzy Sets and Fuzzy Logic," Springer.

[22]K.H.Lee, "First Course on Fuzzy Theory and Applications " Springer, 2005.

[23] عادل عبد النور, "Fuzzy Logic," جامعة الملك سعود.

[24]A. K. S. a. M.Pal, "Triangular fuzzy matrices " Iranian Journal of Fuzzy Systems, vol. 4, no. 1, pp. 75-87, 2007.

[25]M. Omran, "Applications on Some Uncertainty Theories," Master of science in Physics and Engineering Mathematics,Physics and Engineering Mathematics Department, Faculty of Engineering, Tanta University 2016.

[26]Z. Pawlak, "Pawlak Approximation Space and Some of Its Generalizations," 1982. 